\title{
MODELOS DE ESTIMACIÓN DE LA COMPETENCIA Y MORTALIDAD INDIVIDUAL PARA RENOVALES DE ROBLE (Nothofagus obliqua (Mirb)(Oerst)) Y RAULÍ (Nothofagus alpina) (Poepp et Endl))
}

CARLOS BAHAMÓNDEZ VILLARROEL (*) y MARJORIE MARTIN STUVEN (*)

(*) Ingeniero Forestal, Instituto Forestal, Subgerencia de Tecnologías Silvícolas

\section{RESUMEN}

Se proponen modelos para la estimación de la mortalidad individual en renovales de RobleRauli mediante la utilización de un índice de dominancia y la comparación con índices de competencia descritos por otros autores. Se establece el algoritmo de asignación de muerte individual debido a competencia, una expresión para asignación de muerte aleatoria mediante la utilización de la expresión modificada descrita por Botkin(1993).

Palabras clave: Mortalidad, Competencia, Dominancia

\section{ABSTRACT}

Models for the individual mortality and competition are proposed for Nothofagus alpina and Nothofagus obliqua second growth forest A comparison between some historical competition index and the dominance index is carried out. A mortality algorithm of assignation of individual trees dead due competition and a random dead assignation algorithm are described by using $a$ modified approach from Botkin (1993).

Keywords: Mortality, Competition, Dominance. 


\section{INTRODUCCIÓN}

La competencia es un fenómeno característico en individuos sometidos a estrés por recursos. Durante las primeras etapas de desarrollo del individuo la competencia puede no manifestarse sino hasta el momento en que las copas se entrecruzan. No obstante, dependiendo de las condiciones de sitio, la oportunidad de competencia aparece más pronto cuando los sitios son más pobres.

Para el caso de rodales nativos provenientes de regeneración natural como el caso de los renovales de Roble-Raulí, la competencia se presenta en forma dispersa en distintos grados y en distinta oportunidad, dependiendo de la distribución horizontal de los individuos y de su forma de regeneración natural.

La importancia del estudio de la competencia radica en la capacidad de afectar el crecimiento de los individuos debido a la presencia de más individuos de la misma o diferente especie. Merriam (1987), describe la reducción del crecimiento, reducción de la capacidad fotosintética, debilitamiento y reducción de copa como algunos de los principales efectos de la competencia. Este efecto sobre los individuos y sobre el rodal resulta, en su etapa extrema, en la muerte de los individuos. De ahí entonces la importancia del estudio de la competencia como una forma de explicar y/o describir la muerte individual.

El objetivo de estudiar la competencia para renovales de Roble-Raulí consiste en utilizar su efecto como un factor de control del crecimiento modificando la tasa de crecimiento potencial. A este propósito, varios autores han intentado describir la competencia mediante el uso de índices (Newnham y Smith, 1964; Daniels, 1976). La mayoría de los índices descritos se basan en variables de tamaño de los individuos y la distancia entre competidores.

En el desarrollo de la investigación "Investigación Modelos de Simulación para Renovales de Roble Raulí", la competencia se explica mediante el uso de un índice que describe la dominancia relativa del individuo, desarrollado por Bahamóndez y Martin (1992). Este concepto de dominancia o preponderancia fue utilizado por Laroze (1985) para explicar la distribución espacial de Lenga (Nothofagus pumilio). Ambos índices se basan en el tamaño de los individuos y en la distancia entre ellos.

La mortalidad individual se enfoca como una consecuencia de los niveles de competencia alcanzados por los individuos, definiéndose dos tipos de causas de muerte. Aquella derivada de la competencia por recursos y otra debida a causas no identificables. 


\section{DATOS}

Los datos utilizados en el estudio de la mortalidad provienen de parcelas permanentes instaladas en 1991 bajo el proyecto "Investigación Modelos de Simulación para Renovales de Roble-Raulí". Estas parcelas en número de 3, comprenden una extensión de 1 hectárea cada una y 2 de ellas se localizan en la cordillera de los Andes en el límite de la VIII y IX Regiones, frente a la Reserva Nacional Malleco, específicamente en los predios El Morro y Carmen y Maitenes de propiedad de Forestal JCE. Una tercera parcela se ubica en la X Región en el sector del complejo Panguipulli, Nueva Remeco, de propiedad de Forestal Neltume Carranco. El total de árboles muestreados es de 3.962 individuos con rango de edad entre 37 y 65 años.

Cada parcela permanente presenta mayoritariamente Raulí (Nothofagus alpina) y Roble (Nothofagus obliqua) en el dosel superior, y Lingüe (Persea lingüe), Avellano (Gevuina avellana) y Tepa (Laurelia philippiana) en los doseles intermedio e inferior.

En cada parcela permanente se obtiene información de tamaño de cada individuo, DAP, altura total, altura a inicio de copa, diámetro a inicio de copa, diámetro de copa, muestra de edad y posición en plano cartesiano de todos los individuos. Para la estimación de mortalidad se ha realizado un seguimiento de estas parcelas durante 5 años.

\section{MODELOS Y MÉTODOS DE ESTIMACIÓN DE COMPETENCIA Y MORTALIDAD}

\section{Modelos de Estimación de la Competencia}

Los modelos de competencia que se prueban para su uso como modificadores del crecimiento individual, comprenden principalmente aquellos que se basan en el cálculo de la distancia entre individuos como un estimador de las interrelaciones individuo competidor. Se asume individuo competidor a todo aquel individuo que se localice dentro del área de desarrollo de otro individuo generándose interacción negativa.

Los siguientes modelos fueron evaluados para su utilización como modificadores del crecimiento individual. 


\section{Índice de Glover y Hool (1979)}

$$
G=\frac{\pi \frac{D i}{2}}{\pi\left[\left(\left(\sum_{j=1}^{n} D j\right) / n\right) / 2\right]^{2}}=\frac{D^{2}}{\overline{D^{2}}}
$$

donde:

$D_{i}=$ Diámetro a la altura del pecho del individuo " $i "$

$D=$ Diámetro a la altura del pecho del individuo de DAP medio de la parcela.

$n=$ Número de individuos competidores.

Este índice es descrito por Glover y Hool como un índice de área basal independiente de la distancia, representando la razón entre el área basal del árbol " $i$ " y el árbol que representa el DAP medio de la parcela.

Índice de Hegyi (1974)

$$
C_{i j}=\sum_{j=1}^{n}\left(\frac{D_{i}}{D_{j}}\right) /\left(L_{i j}\right)
$$

$D_{i}=$ Diámetro a la altura del pecho del individuo " $j "$

$D_{j}=$ Diámetro a la altura del pecho del competidor "i".

$L_{i j}=$ Distancia entre individuo " $i$ " e individuo " $j$ ".

$n=$ Número de individuos competidores en un radio fijo.

Esta expresión fue descrita por Hegyi basándose en un factor de área basal de 2,3 m², incluyendo como competidores a todos los individuos que clasifiquen dentro del factor. Este índice a diferencia del índice de Glover y Hool depende de la distancia.

\section{Índices de Preponderancia o Dominancia Relativa}

La dominancia de un individuo sobre otro es una medida dependiente de la distancia, cuyo objetivo es cuantificar el grado de influencia que un individuo de tamaño dado es capaz de ejercer sobre sus competidores. Este concepto fue utilizado por Laroze (1985) para aplicarlo a modelos espaciales de Lenga, y utilizado por Bahamóndez y Martin (1992) mediante la descripción de un índice que permite la asignación de un valor de 
dominancia a cada individuo según su tamaño y el de aquellos que lo rodean. A partir de este valor de dominancia es posible también deducir un valor de competencia.

$$
W_{i}=\frac{1}{n} \sum_{j=1}^{n} \frac{1}{\left(d x-d_{i j}\right)} \frac{\left(D_{i}-D_{j}\right)}{\left(D_{i}+D_{j}\right)}
$$

$$
I C\left(W_{i}\right)=1-\left(\frac{W_{i}}{W_{\max }}\right)
$$

donde:

$W_{i} \quad=$ Dominancia del árbol " $i "$

$W_{\max }=$ Dominancia máxima.

$I C\left(W_{i}\right)=$ Competencia del árbol " $i$ " (derivada de la dominancia)

$D_{i} \quad$ = Diámetro (DAP) del árbol objeto " $i "$

$D_{j} \quad$ = Diámetro (DAP) del árbol competidor " $j "$

$D_{i j} \quad=$ Distancia entre árbol objeto y árbol competidor.

$D x \quad=$ Radio máximo de influencia $(4,1 \mathrm{~m})$.

$N=$ Número de individuos competidores en el radio máximo.

\section{Modelos para la Estimación de la Mortalidad Individual.}

La estimación de la mortalidad individual depende de las condiciones de crecimiento individual y del crecimiento de los competidores más cercanos contenidos en el radio fijo $(4,1 \mathrm{~m})$. Para asignar muerte a algún individuo, se establece un seguimiento de las condiciones de crecimiento individual, considerando la relación con el crecimiento de sus vecinos. Esto, como una forma de asignar muerte a aquellos individuos que relativamente se encuentren en niveles muy bajos de crecimiento. La asignación de muerte sobre las bases anteriores describe el fenómeno debido a competencia. Cuando los individuos mueren sin causa identificable, se aplica una función que describe la probabilidad de muerte debida a causas desconocidas.

Asignación de muerte sin causa identificable.

La siguiente expresión describe la función de asignación de muerte sin causa identificable y corresponde a una versión modificada de la proposición de Botkin D. (1993). 


$$
M_{i}=\left(1-\varepsilon_{i}\right)^{E_{i} / E_{\max }}
$$

donde:

$M_{i} \quad=$ Probabilidad que un individuo " $i$ " alcance la edad máxima.

$\xi_{i} \quad=$ Probabilidad anual de muerte para el individuo " $i$ " de la especie de acuerdo a su estado evolutivo.

$E_{i} \quad=$ Edad actual del individuo " $i$ ".

$E_{\text {max }}=$ Edad máxima que sobrevive la especie.

Para este caso los valores de probabilidad de supervivencia $M_{i}$ se calculan considerando una edad máxima de 120 años.

Muerte individual debida a competencia por recursos

La muerte de individuos causada por competencia se explica a través de la observación del comportamiento del incremento en diámetro durante un período de proyección. La muerte individual se asigna cuando la suma de los incrementos de los individuos competidores alcanza el diámetro del individuo objeto. Se utiliza el diámetro como indicador, debido a la sensibilidad de esta variable a la densidad. Este algoritmo permite seleccionar los individuos para muerte de acuerdo a las condiciones específicas de competencia que dependen del micrositio en que se encuentran los árboles. Luego, si los individuos en total, dentro del radio fijo, se encuentran en condiciones marginales, sus tasas de crecimiento serán bajas con relación a otras partes del rodal, por lo tanto una asignación de muerte sólo por el criterio de nivel de incremento mínimo, asignaría muerte a todo un grupo de individuos, errando en la evaluación real de la competencia. Este enfoque permite describir con más realidad la situación de menoscabo de un individuo de acuerdo a la competencia por recursos disponibles en su entorno directo y cualquier cambio en las condiciones de ese entorno significará un cambio en la selectividad individual para muerte. Un raleo, por ejemplo, favorecerá las condiciones de sobrevivencia individual retrasando la asignación de muerte.

\section{Estimación}

Para seleccionar el índice de competencia (dominancia) más adecuado se establece un análisis de correlación entre cada índice y los incrementos diamétricos. Esto se basa en el cálculo del coeficiente de correlación de Pearson. Cuya expresión es: 


$$
r=\frac{S_{x y}}{S_{x} S_{y}}
$$

donde

$$
\begin{aligned}
& S_{x y}=\frac{\sum\left(x_{i}-\bar{x}\right)\left(y_{i}-\bar{y}\right)}{(n-1)} \\
& S_{x}=\sqrt{\sum\left(x_{i}-\bar{x}\right)^{2} /(n-1)} \\
& S_{y}=\sqrt{\sum\left(y_{i}-\bar{y}\right)^{2} /(n-1)}
\end{aligned}
$$

con:

$S_{x y}=$ Covarianza de $\boldsymbol{x}$ e $\boldsymbol{y}$

$S_{x}=$ Desviación estándar de $\boldsymbol{x}$.

$S_{y}=$ Desviación estándar de $\boldsymbol{y}$.

Para el caso de la mortalidad individual, la estimación de muerte a nivel de rodal es el resultado de la suma de individuos con muerte asignada cada período. Luego, depende directamente del comportamiento del crecimiento y de la evaluación de la competencia como modificador del crecimiento. Para el caso de muerte sin causa identificable, la muerte se asigna mediante el cálculo de un número aleatorio para cada individuo. Si este número es inferior a la probabilidad calculada de supervivencia $\boldsymbol{M}_{\boldsymbol{i}}$, entonces se asigna muerte.

\section{AJUSTE DE MODELOS Y RESULTADOS}

\section{Modelos de Competencia}

Los modelos de competencia descritos por (1), (2) y (3) fueron calculados sobre la base de las parcelas 1 y 2 del sector de VIII y IX Regiones. Se calcula la correlación de cada índice con los incrementos diametrales y se selecciona un índice como el apropiado para ser utilizado como modificador del crecimiento. 
El Cuadro 1 describe la correlación entre incremento diametral y competencia para índices de Glover-Hool, Hegyi y Bahamóndez-Martin.

\section{Cuadro 1}

\section{CORRELACIÓN PARA DISTINTOS ÍNDICES DE COMPETENCIA.}

\begin{tabular}{|c|l|c|}
\hline Modelo & Índice & $\mathrm{r}$ (Pearson) \\
\hline 1 & Bahamóndez-Martin & 0,400 \\
\hline 2 & Glover-Hool & 0,165 \\
\hline 3 & Hegyi & 0,168 \\
\hline
\end{tabular}

Las correlaciones son más altas en el modelo $\mathrm{N}^{\circ} 1$, por lo que se estima como una buena aproximación de la Dominancia relativa del individuo sobre los demás. Este índice puede entonces ser incluído como elemento modificador del crecimiento permitiendo una mejor estimación del crecimiento en diámetro. Los modelos $\mathrm{N}^{\circ} 2$ y $\mathrm{N}^{\circ} 3$ del cuadro $\mathrm{N}^{\circ} 1$ presentan bajas correlaciones aunque similares. Esta semejanza puede deberse a que ambos índices aproximan bien el área basal del individuo presentándolo como un efecto resultante de la competencia. Sin embargo, y a pesar que el área basal está relacionada con la ocupación del sitio, otros elementos como cambios en los recursos disponibles debido a micro - variaciones del entorno inmediato, no son bien expresados por estos índices. El modelo $\mathrm{N}^{\circ} 1$, presenta mejor correlación debido a que refleja situaciones de competencia por recursos, utilizando las diferencias entre diámetros, número de individuos y distancia entre competidores, como estimadores de las condiciones del entorno cercano a los individuos. Así, una gran diferencia entre un individuo e individuo competidor (se supone homogeneidad en edades) refleja una fuerte escasez de recursos en el micrositio.

La competencia para individuos se calcula basado en la expresión de dominancia del modelo $\mathrm{N}^{\circ} 3$, utilizándose como modificador del crecimiento potencial en la forma siguiente:

$$
d x / d t=\delta+\frac{\alpha}{(1+\exp (\beta-\gamma X))}
$$

donde:

$\alpha, \beta, \delta$ y $\gamma=$ Coeficientes a estimar.

$\boldsymbol{d x} / \boldsymbol{d} \boldsymbol{t}=$ Variable a estimar, (Incremento en DAP).

$\boldsymbol{X}=$ Variable independiente $\left(X=D A P^{*} W\right)$

W = Indice de Dominancia.

DAP = Diámetro a la altura del pecho. 
Este modelo de crecimiento descrito para renovales de Roble - Raulí, utiliza una variable transformada $X=D A P^{*} W$ para la estimación del incremento en diámetro. Esta transformación permite al índice de dominancia modificar el crecimiento de acuerdo a valores de dominancia.

\section{Mortalidad}

Para la estimación de la mortalidad individual sin causa identificable, la función (5) se ejecuta mediante el siguiente algoritmo:

1. Cálculo de la probabilidad anual de muerte de acuerdo a la edad del individuo.

2. Cálculo de la probabilidad de alcanzar la edad máxima para ese individuo.

3. Cálculo de números aleatorios para asignación a cada individuo.

4. Si el número aleatorio asignado al individuo resulta menor que el valor calculado en 2 , se asigna muerte al individuo.

Este algoritmo permite una buena aproximación a la mortalidad debida a causas distintas a la competencia, y se ejecuta cada año de proyección del crecimiento individual. La figura 1 muestra el comportamiento de la curva de probabilidad de supervivencia según la edad.

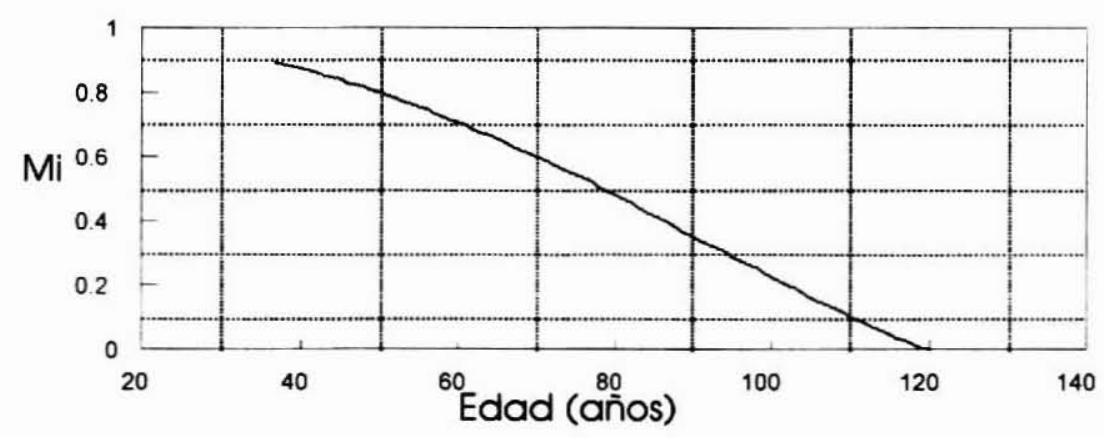

Figura 1. PROBABIIDAd DE SUPERVtVEnCLA DE ACUERDO A EDAD MAXMMA

Asignación de muerte debida a competencia

La asignación de muerte por competencia se realiza por seguimiento de los valores de incremento diametral durante el período de proyección. El algoritmo al inicio de la 
proyección del crecimiento establece una lista de mortalidad para cada individuo, esta lista consiste en la acumulación del crecimiento diametral durante el período de proyección y el registro del valor de Dominancia (W). Si la suma de la acumulación del crecimiento de los individuos competidores iguala o supera el diámetro del individuo objeto, y su Dominancia se ha mantenido negativa durante todo el período de acumulación, entonces se asigna muerte.

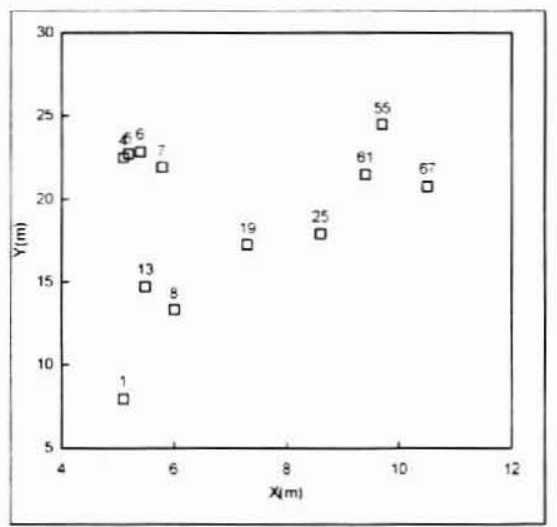

Figura 2. UBICACión EsPacial de LOS INDIVIDUOS EN PLANO CARTESIANo.

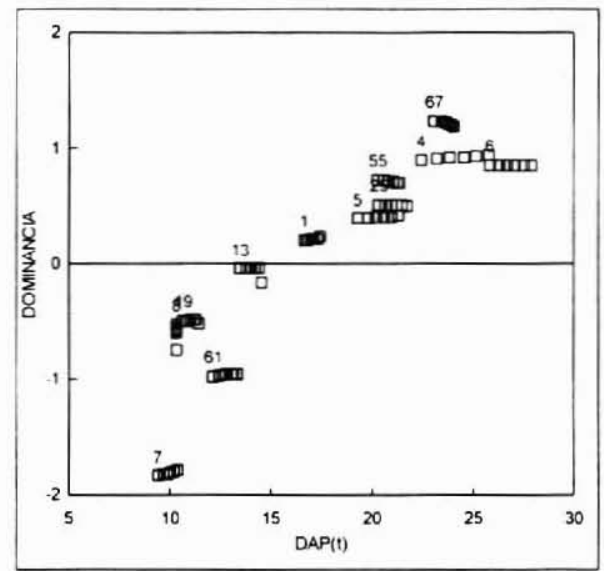

Figura 3. COMPORTAMIENTO DE LA DOMINANCIA DE ACUERDO AL CRECIMIENTO EN DIAMETRO. 
Las figuras 2 y 3 describen una pequeña área de la parcela permanente 2. En la figura 2 es posible observar el comportamiento del incremento en diámetro y su relación con el valor de Dominancia (W). Se observan árboles cuyo crecimiento se mantiene parejo y positivo, de pendiente ascendente, como es el caso de los individuos $1,4,5,6,25$, éstos se encontrarían de acuerdo al gráfico en pleno crecimiento y con espacio disponible no competido. No obstante lo anterior, la figura 2 muestra que la posición de los individuos 4,5,6 y 7 es muy cercana y su crecimiento debería verse afectado en forma considerable. Esto sugiere que este grupo de árboles está siendo favorecido por condiciones ajenas a la competencia, como condiciones de micrositio, o posición espacial favorecida por microrelieve que permiten mejor acceso a recursos de luz y nutrientes.

Por otra parte, existen individuos que aunque presentan valores de dominancia positivos, y una tasa de crecimiento en diámetro constante, la pendiente de la curva descrita durante el período de crecimiento muestra una tendencia negativa, como es el caso de los individuos 67 y 55, esto indica la presencia de un efecto negativo de competencia sobre los individuos provocado por la presencia de otros, en este caso sería el individuo 61 cuya pendiente es notoriamente creciente estableciéndose como el elemento perturbador. Otro caso es el de aquellos individuos cuya pendiente es definitivamente negativa e incluso nula, ejemplo los árboles 8 y 19 de la figura 3 . En la figura 2 se observa que ambos individuos son competidores dada su posición espacial, en este caso específico el árbol 8 de permanecer en esas condiciones de crecimiento por un período prolongado, morirá. Este último caso es el que describe la forma en que funciona el algoritmo de mortalidad implementado.

\section{REFERENCIAS}

Bahamóndez C., Martin M., Kahler C., Blanco R. 1992. Investigación Modelos de Simulación Renovales de Roble Raulí. Etapa preliminar. Informe final.

Botkin D. 1993. Forest Dynamics an Ecological Model. Oxford University Press.

Daniels R. ; Burkhart, H.1986. A comparison of competition measures for predicting growth of loblolly pines trees. Canadian journal of forest research. Vol 16, No 4, pp 1230-1237.

Laroze A. 1985. Modelo de Estructura Espacial para Rodales Puros de Lenga. Tesis U. Austral de Chile. Fac.de Cs. Forestales Valdivia.Chile.

Merriam, R. 1987. A generalized competition index for Sric stands. Forest growth modelling and prediction. USDA Forest Services. Northern Central Forest Exp.Stn.General Tech.Report NC-120 pp $110-117$.

Newnham, R.; Smith J.H.G.1964. Development and testing stand of stands models for Douglas fir and Lodgepole pine. Forestry chronicles $\mathrm{N}^{\circ} 40$ pp 452-502. 